\title{
Effect of Individual Properties of Flexible Chainlike Walkers in a Many- Body System
}

\author{
Takashi Mashiko*
}

Department of Mechanical Engineering, Shizuoka University, Johoku 3-5-1, Naka-ku, Hamamatsu, Japan

\begin{abstract}
Several versions of the "flexible chainlike walker" (FCW) model are proposed and investigated by numerical simulations on a square lattice. It is shown that the "original" FCWs aggregate spontaneously and irreversibly, where no adherence is assumed, triggered by "mutual locking". This is in complete contrast to established aggregation models which necessarily require adherence, and exemplifies the significance of the deformability of self-driven objects. The behaviors of the "smart" FCWs, which have higher ability of moving, are similar to those of the original FCWs. This suggests that the spontaneous, irreversible aggregation is a robust feature peculiar to a many-body system of deformable self-driven objects. On the other hand, the "double-headed" FCWs do not undergo the mutual locking and the resultant irreversible aggregation, unlike the original and the smart FCWs. This indicates that "bidirectionality" prevents deformable self-driven objects from aggregating.
\end{abstract}

Keywords: Aggregation, deformable objects, random walk.

\section{INTRODUCTION}

Dynamics of systems where numerous self-driven objects are concerned is one of the hottest topics in the field of multidisciplinary physics. Various systems have been modeled mainly based on the random-walk model, and examined mainly by numerical simulations. For example, the territory visited by many random walkers $[1,2]$ and aggregation processes of numerous random walkers [3-9] have been investigated. Also, traffic flows have been modeled by using the "biased" random-walk models [10-17]. The usefulness and the wide applicability of the randomwalk model are obvious. In addition, many related models have been considered, where the walker is deformable or affected by its own track. For instance, the reptation model [18], self-avoiding walk (SAW), true SAW (TSAW) [19], indefinitely-growing SAW (IGSAW) [20], smart kinetic walk (SKW) [21], Laplacian random-walk (LRW) [22,23], and the kinetic growth walk (KGW) [24] have been proposed, and their behaviors have been investigated and contrasted. While the dynamics of a single self-driven object has been deeply studied with these models, however, in studying many-body systems of self-driven objects, each object has been treated just as a point-like particle [3-7,10$12]$ or, at most, as a rigid body $[8,9,13-17]$ which does not possess deformability.

From the point of view of statistical physics, it is important and intriguing to examine how the microscopic, individual properties of the self-driven object affect the macroscopic, collective behaviors. Also, from the standpoint of application potentiality, it is interesting to investigate the properties of deformable self-driven objects, since there are actually various self-driven objects that are deformable

\footnotetext{
*Address correspondence to this author at Department of Mechanical Engineering, Shizuoka University, Johoku 3-5-1, Naka-ku, Hamamatsu, Japan; E-mail: ttmasik@ipc.shizuoka.ac.jp
}

around us. Even for one-dimensional objects alone, examples can be given from those living, such as some kinds of bacteria and worms, to those nonliving, such as container dollies towed by a tractor and snake-like robots. Especially, studying the behaviors of deformable machines is of increasing importance, in view of the rapid factory automation and the recent development of snake-like robots [25]. Therefore, it is a worthwhile issue to study the effect of the deformability of self-driven objects. Recently, the author proposed the "flexible chainlike walker" (FCW) model as a minimal model of deformable self-driven objects and as an extension of the regular random-walk model, and reported spontaneous, irreversible aggregation without adherence as a unique phenomenon peculiar to a many-body system of deformable self-driven objects [26]. In this paper, two extended versions of the model are proposed in the hope of a further understanding of many-body systems of deformable self-driven objects. Also, the FCW model has shown application potentiality to the study of some kinds of transport system or traffic flow, when "bias" is introduced $[27,28]$. This suggests that extending the FCW model also contribute to possible future applications in transport systems, though direct modeling of certain entities is not intended in this paper.

In what follows, the original FCW model and the irreversible aggregation process are briefly reviewed first. Then two derivative versions, the "smart" and the "doubleheaded" FCW models, are introduced and compared with the original model.

\section{MODEL}

The basic idea of the FCW model is as follows: An FCW of length $l$ is represented by $l$ serially-concatenated particles, which, on a square lattice, occupy $l$ horizontallyor vertically-adjacent sites. One of the edge particles (the first particle) represents the head of the FCW and the other (the $l$ th particle) represents the tail. Fig. (1) schematically 
exemplifies the movement of an $l=5 \mathrm{FCW}$, where the head particle is represented by the double circle. At each time step, the head particle can choose and move to one of the nearest neighbor sites that is not occupied by another particle (either of the same FCW or of another). Then the subsequent particles follow the head particle. That is, the second particle moves to the site that the head particle has just left, the third particle moves to the site that the second particle has just left, and so forth. If the head particle fails to choose an unoccupied nearest neighbor site, the FCW does not move at that time step.
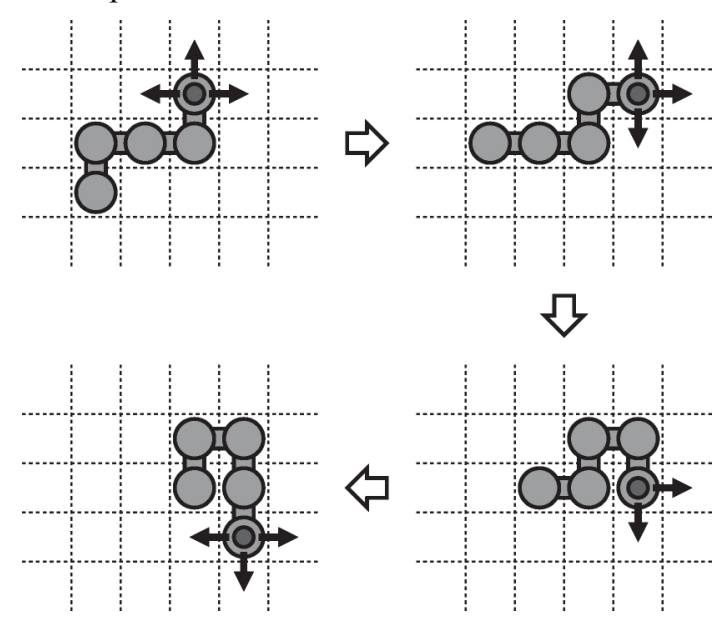

Fig. (1). Flexible chain-like walker model on a square lattice. A typical series of movements of an $l=5 \mathrm{FCW}$ is illustrated. At each time step, the head particle (represented by the double circle) can choose and move to an unoccupied nearest neighbor site in a random manner, followed by the subsequent particles. Possible moving directions are indicated by the arrows. (See text for details.)

As shown above, the FCW model is a simple model, which has a single free parameter, namely the length $l$. Also, the regular random-walk, in which the point-like walker moves to one of the nearest neighbor sites at every time step, irrespective of its track, is recovered from the FCW model in the limit of $l=1$. Thus the FCW model is an extension of the regular random-walk model. It should be noted that an FCW of $l \geq 3$ is deformable, while an FCW of $l=2$ is a rigid body which does not change shape but just changes direction.

Now, several versions of the FCW model can be suggested depending on the specific way of FCW's moving: In the original FCW model [26], the head particle chooses one of its four nearest neighbor sites at random at each time step and moves to that site if it is vacant. If the chosen site happens to be an occupied one, the FCW does not move. On the other hand, in the smart FCW model, the head particle always chooses and moves to an unoccupied nearest neighbor site unless impossible (i.e., except when all the four are occupied). Thus the smart FCW is expected to have higher possibility of moving in comparison with the original FCW. Also, another derivative, the double-headed FCW model, can be introduced. In this model, the head and the tail particles of each FCW are not fixed but switch with each other randomly at every time step, being reminiscent of "pushmi-pullyu", a fictional creature in "The Story of Doctor Dolittle" [29]. The randomly-selected head particle chooses one of its four nearest neighbor sites at random and moves to that site if it is vacant, followed by the subsequent particles, as in the case of the original FCW (not the smart FCW).

\section{RESULTS AND DISCUSSION}

In the present study, $N$ FCWs are placed on a square area of $W \times W$ sites. To describe the behaviors of FCWs, the following quantities are defined: The density of particles is the ratio of the total number of particles to the number of sites on the square area; $\rho=l N / W^{2}$. The mobility of FCWs at time $t$ is defined as $M(t)=N_{\text {mov }}(t) / N$, where $N_{\text {mov }}(t)$ is the number of FCWs that have succeeded in moving at that time step. At $t=0$, FCWs are placed at random positions as initial distribution. Each FCW is put straight horizontally. This is partly for simplicity and partly for avoiding inborn "locking" (explained below). Then the FCWs are updated following the above-described rules in random order at every time step. In the present work, simulations are conducted with $W=100$ under periodic boundary condition.

The following are the results of the simulations. First, typical results for the original FCWs [26] should be mentioned. It was found that the mobility $M$ decreases with time, to an asymptotic value $M_{\infty}=M(t \rightarrow \infty)$. Typically, $t \sim 50,000$ was enough for $M$ to reach $M_{\infty}$. In Fig. (2) the values of $M_{\infty}$, each of which is averaged over 50,000 time steps in the asymptotic state (typically, $t=50,001-100,000$ ) and over at least 10 runs, are plotted as functions of $\rho$ for $1 \leq l \leq 8$. Whereas the $M_{\infty}-\rho$ curves for $l=1$ and 2 are linear (which were shown to agree with the theoretical formulas of $M_{\infty}=1-\rho$ and $M_{\infty}=\frac{3}{4}(1-\rho)$, respectively), those for $l \geq 3$ are qualitatively different. The curves for $l=3$ and 4 show a sharp drop from a higher- $M_{\infty}$ state in the lower- $\rho$ region to an $M_{\infty} \sim 0$ state in the higher- $\rho$ region. Furthermore, for $l \geq 5, M_{\infty}$ remains nearly 0 in the whole $\rho$-region investigated. It was demonstrated that when the density $\rho$ is higher the deformable FCWs (i.e., $l \geq 3$ ) come to get immobile, with their head particles surrounded by each other, which the author named "mutual locking", resulting in the formation of aggregates. Some of the mutual-locking patterns are exemplified in Fig. (3). Discussion about the mutual locking was able to qualitatively explain the basic features observed in Fig. (2). (These results for original FCWs are detailed in Ref. [26]).

Now, the results for the smart FCWs are shown in Fig. (4), corresponding to Fig. (2). It is clear that, for $l=1$ and 2 , the $M_{\infty}-\rho$ curves are not linear, and the mobility $M_{\infty}$ of the smart FCWs is higher than that of the original FCWs at the same density $\rho$. However, the smart FCWs give similar results to those of the original FCWs when they are deformable. For $l=3$ and 4 , the $M_{\infty}-\rho$ curves show the transition from the moving (higher- $M_{\infty}$ ) state to the aggregating $\left(M_{\infty} \sim 0\right)$ state. And the values of the critical density at which the transition occurs are nearly the same, as 
can be seen in the closeup of Fig. (5), though the $M_{\infty}$-values in the moving state are higher than those of the original FCWs. For $l \geq 5, M_{\infty}$ remains nearly 0 as in the case of the original FCWs.

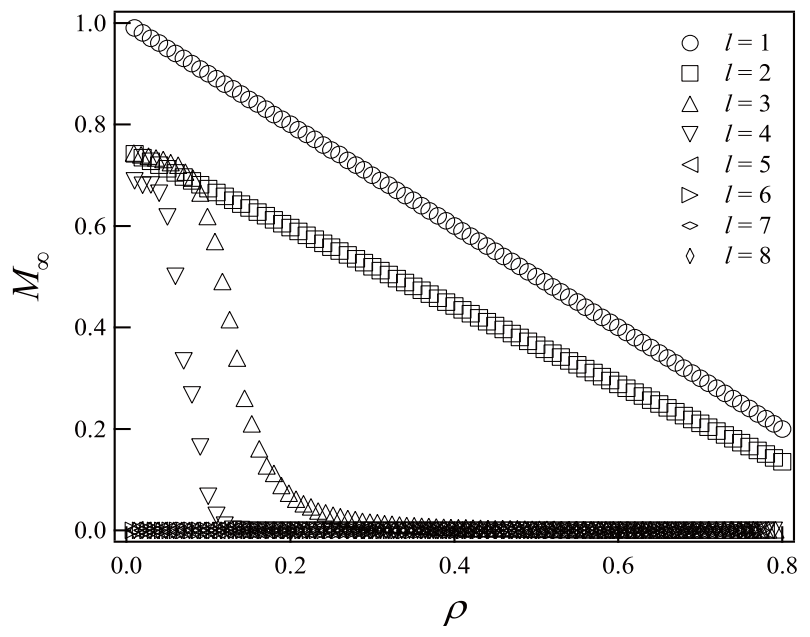

Fig. (2). Asymptotic mobility $M_{\infty}$ as a function of particle density $\rho$ for $1 \leq l \leq 8$ original FCWs. Each symbol represents the averaged value taken over 50,000 time steps in the asymptotic state and over at least 10 runs. (a)

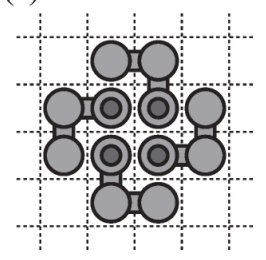

(b)

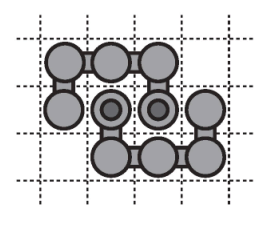

(c)

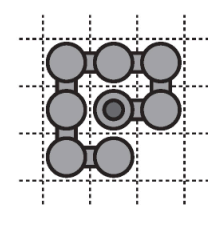

Fig. (3). Examples of mutual locking of FCWs. (a) Four-body mutual locking of $l=3$ FCWs. (b) Two-body mutual locking of $l=5$ FCWs. (c) Self-locking of an $l=8$ FCW. (See Ref. [26] for details).

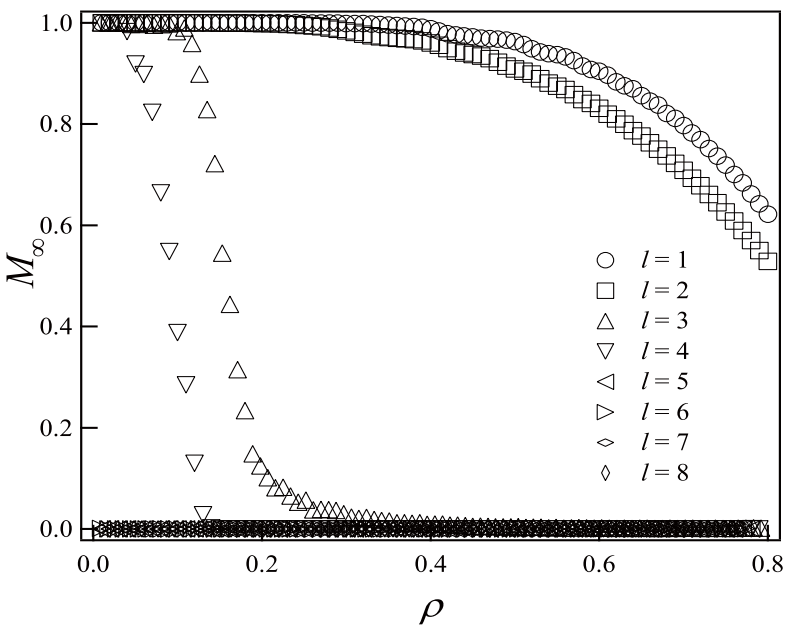

Fig. (4). Asymptotic mobility $M_{\infty}$ as a function of particle density $\rho$ for $1 \leq l \leq 8$ smart FCWs. Each symbol represents the averaged value taken over 50,000 time steps in the asymptotic state and over at least 10 runs.

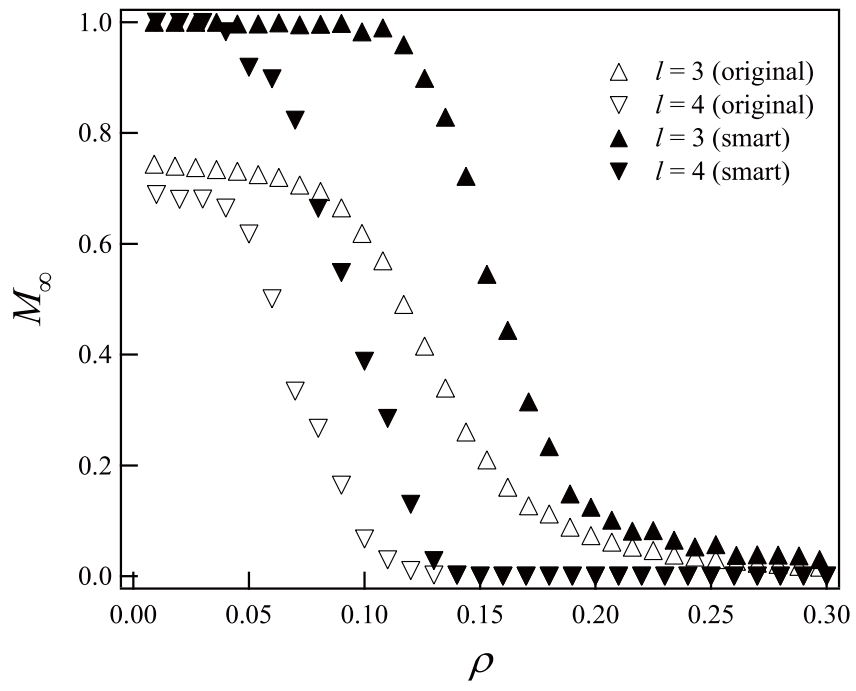

Fig. (5). Closeup of the $M_{\infty}-\rho$ curves of the original and the smart FCWs near the transition points for $l=3$ and 4 .

The fact that the qualitative behaviors are the same for the original and the smart FCWs means that the introduction of the "smartness" into each FCW does not essentially affect the collective behaviors. It can be concluded that the occurrence of the mutual locking and the resultant irreversible aggregation are robust features of a many-body system of unidirectional, deformable, self-driven objects represented by the original or the smart FCW model.

Here, it is worth mentioning the novelty and the essence of the aggregation mechanism observed in the systems of the original and the smart FCWs. There have been several models of aggregation processes; from those of point-like particles [3-7,30-32] to those of particles aggregates [8,9,33]. In all these established models some adherence has been assumed. That is, in terms of a particle-aggregation process like the well-known diffusion-limited aggregation (DLA) [4] or ballistic deposition [30,32], a particle adheres to and becomes part of an existing aggregate when it comes to contact the aggregate, even if stochastically (i.e., with a sticking probability $p>0$ ). Also, it is easy to see that adherence is virtually assumed in somewhat different types of aggregation models like the Eden model [34], which was originally proposed for the growth process of a cell colony, where one of the adjoining sites of the colony (aggregate) is chosen and occupied by a new cell (particle). Obviously, in all these models adherence is essential for the aggregation process and the irreversibility is attributable to this adherence. In the present models, on the other hand, FCWs aggregate spontaneously and irreversibly, though no such adherence is assumed (i.e., $p=0$ ). Adherence is not required for the occurrence of the aggregation of FCWs. Therefore, the aggregation mechanism is fundamentally different from that of the conventional models. The fact that the aggregation is observed only for $l \geq 3$ clearly indicates that the occurrence and irreversibility of the aggregation process are brought about by the deformability of FCWs, via mutual locking.

Next, the results for the double-headed FCWs are shown in Fig. (6), corresponding to Figs. $(\mathbf{2}, \mathbf{4})$. The $M_{\infty}-\rho$ curve 
for $l=1$ agrees with that of the original FCWs (i.e., $\left.M_{\infty}=1-\rho\right)$. This is quite expected, because in this case each FCW is a point-like particle (or the regular randomwalker) and there is no point in considering the head and the tail particles; that is, the double-headed FCW is identical with the original $\mathrm{FCW}$ for $l=1$. On the other hand, the results for $l \geq 2$ are different from those of the original FCWs. The $M_{\infty}-\rho$ curve for $l=2$ is convex upward, while that of the original FCWs was a straight line as in the case of $l=1$. What is more important, however, are the results for the deformable FCWs. The curves for $l=3$ and 4 show no transition from a higher- $M_{\infty}$ state to an $M_{\infty} \sim 0$ state. Instead, they are similar to that of $l=2$ and approach to the straight line of $l=1$ as the density $\rho$ increases. In other words, the double-headed FCWs do not undergo the irreversible aggregation but keep higher mobility. The difference between the original or the smart FCWs and the double-headed FCWs is obvious. While in the former case the $M_{\infty}$-gap between $l=1$ and $l=3$ or 4 widens due to the transition, in the latter case it narrows monotonically as $\rho$ increases. Also, while in the former case the $M_{\infty}$-values for $l \geq 5$ remain nearly 0 , in the latter case they are very close to that for $l=2$ and keep a high level in the whole $\rho$ region investigated.

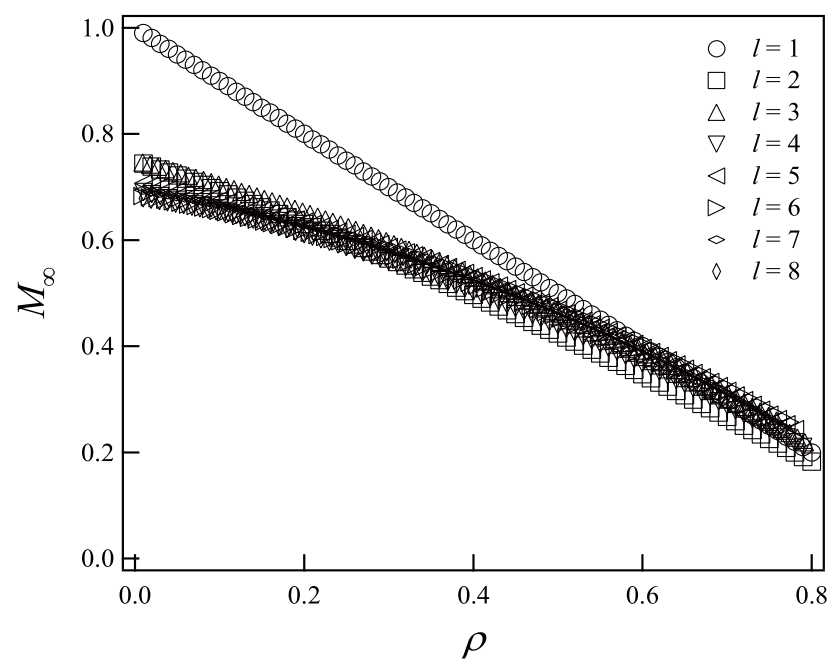

Fig. (6). Asymptotic mobility $M_{\infty}$ as a function of particle density $\rho$ for $1 \leq l \leq 8$ double-headed FCWs. Each symbol represents the averaged value taken over 50,000 time steps in the asymptotic state and over at least 10 runs.

The system of the double-headed FCWs is characterized also by the fact that all the $M_{\infty}-\rho$ curves (of $l \geq 2$ ) are close to each other, which means that the length $l$ does not much affect the collective behaviors. This is in contrast with the cases of the original and the smart FCWs, where the length acts as an "immobilizer" in the sense that the mobility $M_{\infty}$ considerably decreases as the length $l$ increases, leading to more opportunities of mutual locking. The reason why the double-headed FCWs do not undergo mutual locking is not because they remain in nearly the same positions, without moving effectively, due to the random switching of the head and the tail particles. In a macroscopic time scale, each double-headed FCW surely travels, as does the regular random-walker whose displacement is proportional to the square root of the elapsed time $(\sqrt{t})$. Nevertheless, the double-headed FCWs do not fall into the $M_{\infty} \sim 0$ state (which was confirmed in long runs of simulation for $t=100,000,000)$.

The comparison of the behaviors of the double-headed FCWs with those of the original or the smart FCWs shows that the introduction of "bidirectionality" into each FCW makes a significant change in the collective behaviors. It can be concluded that bidirectionality prevents deformable selfdriven objects from undergoing the mutual locking and the resultant irreversible aggregation, which are robust features of unidirectional, deformable, self-driven objects.

\section{CONCLUSION}

To conclude, in the present paper, "flexible chain-like walker" (FCW) models have been proposed and their behaviors have been investigated through numerical simulations. In the many-body system of the "original" FCWs, the mutual locking and the resultant irreversible aggregation were observed for $l \geq 3$. They were observed also for the system of "smart" FCWs, where each FCW moves whenever possible. This shows that they are robust, unique characteristics of unidirectional, deformable, selfdriven objects. On the other hand, the "double-headed" FCWs did not aggregate irreversibly as in the cases of the original and the smart FCWs. This indicates that bidirectionality prevents self-driven objects from experiencing the mutual locking and thus the irreversible aggregation.

Further studies of the many-body systems of FCWs, motivated by the present work, would be challenging. For example, extending the FCW model for off-lattice or higherdimensional space may reveal some other properties peculiar to many-body systems of deformable self-driven objects. And, of course, it is intriguing to investigate traffic flows with the use of "biased" FCW models, from the viewpoint of transport phenomena, some of which has actually been done and reported elsewhere $[27,28]$.

\section{REFERENCES}

[1] H. Larralde, P. Trunfio, S. Havlin, H. E. Stanley, and G. H. Weiss, "Territory covered by $N$ diffusing particles," Nature, vol. 355, pp. 423-426, January 1992.

[2] E. Arapaki, P. Argyrakis, and A. Bunde, "Diffusiondriven spreading phenomena: The structure of the hull of the visited territory,” Phys. Rev. E, vol. 69, p. 031101, March 2004.

[3] H. B. Rosenstock, and C. L. Marquardt, "Cluster formation in twodimensional random walkers: Application to photolysis of silver halides," Phys. Rev. B, vol. 22, pp. 5797-5809, December 1980.

[4] T. A. Witten, Jr., and L. M. Sander, "Diffusion-limited aggregation, a kinetic critical phenomenon," Phys. Rev. Lett., vol. 47, pp. 14001403, November 1981.

[5] P. Meakin, "Diffusion-controlled cluster formation in two, three, and four dimensions," Phys. Rev. A, vol. 27, pp. 604-607, January 1983.

[6] M. Matsushita, and H. Kondo, "Diffusion-limited aggregation with tunable lattice anisotropy," J. Phys. Soc. Jpn., vol. 55, pp. 24832486, August 1986.

[7] M. Matsushita, K. Honda, H. Toyoki, Y. Hayakawa, and H. Kondo, "Generalization and the fractal dimensionality of diffusion-limited 
aggregation,” J. Phys. Soc. Jpn., vol. 55, pp. 2618-2626, August 1986.

[8] P. Meakin, "Formation of fractal clusters and networks by irreversible diffusion-limited aggregation," Phys. Rev. Lett., vol. 51, pp. 1119-1122, September 1983.

[9] M. Kolb, R. Botet, and R. Jullien, "Scaling of kinetically growing clusters," Phys. Rev. Lett., vol. 51, pp. 1123-1126, September 1983.

[10] M. Muramatsu, T. Irie, and T. Nagatani, "Jamming transition in pedestrian counter flow," Physica A, vol. 267, pp. 487-498, May 1999.

[11] M. Muramatsu, and T. Nagatani, "Jamming transition in twodimensional pedestrian traffic," Physica A, vol. 275, pp. 281-291, January 2000.

[12] S. Maniccam, "Traffic jamming on hexagonal lattice," Physica A, vol. 321, pp. 653-664, April 2003.

[13] R. Jiang, and Q.-S. Wu, "The moving behavior of a large object in the crowds in a narrow channel," Physica A, vol. 364, pp. 457-463, May 2006.

[14] R. Jiang, and Q.-S. Wu, "Interaction between vehicle and pedestrians in a narrow channel," Physica A, vol. 368, pp. 239-246, August 2006.

[15] R. Nagai, and T. Nagatani, "Jamming transition in counter flow of slender particles on square lattice," Physica A, vol. 366, pp. 503512, July 2006.

[16] M. Fukamachi, and T. Nagatani, "Sidle effect on pedestrian counter flow," Physica A, vol. 377, pp. 269-278, April 2007.

[17] S. Ito, T. Nagatani, and T. Saegusa, "Volatile jam and flow fluctuation in counter flow of slender particles," Physica A, vol. 373, pp. 672-682, January 2007.

[18] P. G. de Gennes, "Reptation of a polymer chain in the pressure of fixed obstacles," J. Chem. Phys., vol. 55, pp. 572-579, July 1971.

[19] D. J. Amit, G. Parisi, and L. Peliti, "Asymptotic behavior of the "true” self-avoiding walk," Phys. Rev. B, vol. 27, pp. 1635-1645, February 1983.

[20] K. Kremer, and J. W. Lyklema, "Indefinitely growing self-avoiding walk," Phys. Rev. Lett., vol. 54, pp. 267- 269, January 1985.
[21] A. Weinrib, and S. A. Trugman, "A new kinetic walk and percolation perimeters," Phys. Rev. B, vol. 31, pp. 2993-2997, March 1985.

[22] J. W. Lyklema, C. Evertsz, and L. Pietronero, "The laplacian random walk," Europhys. Lett., vol. 2, pp. 77-82, July 1986.

[23] J. W. Lyklema, and C. Evertsz, "Reflecting and absorbing boundary conditions on the tail of the laplacian random walk," $J$. Phys. A, vol. 19, pp. L895- L900, October 1986.

[24] I. Majid, N. Jan, A. Coniglio, and H. E. Stanley, "Kinetic growth walk: A new model for linear polymers," Phys. Rev. Lett., vol. 52, pp. 1257-1260, April 1984.

[25] A. A. Transeth and K. Y. Pettersen, "Developments in snake robot modeling and locomotion", in 9th International Conference on Control, Automation, Robotics, and Vision, 2006, pp. 1393-1400.

[26] T. Mashiko, "Irreversible aggregation of flexible chainlike walkers without adherence," Phys. Rev. E, vol. 78, p. 011106, July 2008.

[27] T. Mashiko, Y. Imanishi, R. Kuwajima, and T. Nagatani, "Hardening by softening in a flow of chainlike self-driven objects," Physica A, vol. 388, pp. 3202- 3212, August 2009.

[28] A. Matsui, T. Mashiko, and T. Nagatani, "Traffic flow of mobile objects through obstacles: Turning and translational objects," Physica A, vol. 388, pp. 157- 173, January 2009.

[29] H. Lofting, The Story of Doctor Dolittle. New York: Frederick A Stokes, 1920.

[30] M. J. Vold, "Computer simulation of floc formation in a colloidal suspension," J. Colloid Sci., vol. 18, pp. 684-695, September 1963.

[31] H. P. Hutchinson, and D. N. Sutherland, "An open-structured random solid," Nature, vol. 206, pp. 1036- 1037, June 1965.

[32] P. Meakin, P. Ramanlal, L. M. Sander, and R. C. Ball, "Ballistic deposition on surfaces," Phys. Rev. A, vol. 34, pp. 5091-5013, December 1986.

[33] D. N. Sutherland, "Chain formation of fine particle aggregates," Nature, vol. 226, pp. 1241-1242, June 1970.

[34] M. Eden, Proceedings of the Fourth Berkeley Symposium on Mathematical Statistics and Probability. University of California Press: Berkeley, 1961, vol. 4, pp. 223-239.

(C) Takashi Mashiko; Licensee Bentham Open.

This is an open access article licensed under the terms of the Creative Commons Attribution Non-Commercial License (http://creativecommons.org/licenses/by-nc/ $3.0 /$ ) which permits unrestricted, non-commercial use, distribution and reproduction in any medium, provided the work is properly cited. 